\title{
Surface Micro/Nano-Textured Hybrid PEDOT:PSS-Silicon Photovoltaic Cells Employing Kirigami Graphene
}

Chi-Hsien Huang ${ }^{\dagger *}$, Zih-Yang Chen ${ }^{\ddagger}$, Chi-Ling Chiu ${ }^{\ddagger}$, Tzu-Ting Huang ${ }^{\dagger}$, Hsin-Fei Meng§, and Peichen $\mathrm{Yu}^{\ddagger *}$

'Department of Materials Engineering, Ming Chi University of Technology, New Taipei City 24301, Taiwan

Department of Photonics, College of Electrical and Computer Engineering, National Chiao Tung University, Hsinchu 30010, Taiwan

\$Institute of Physics, National Chiao Tung University, Hsinchu 30010, Taiwan

*E-mail: chhuang@mail.mcut.edu.tw, yup@faculty.nctu.edu.tw

The Supporting Information includes the followings:

The procedures to prepare the inverted pyramid array. The schematic of kirigami pattern with a cross array. Scheme and PV characteristics of hybrid PV cell with the optimized condition for the inverted pyramid structure. PV characteristics of hybrid PV cell with various spin speeds for PEDOT:PSS coating on the inverted pyramid structure. The SEM image of PEDOT:PSS coating on inverted pyramid 
structure at $6000 \mathrm{rpm}$ of spin speed. The reflectance spectra and SEM images of inverted pyramid structure before and after optimized re-etching condition and PEDOT:PSS coated on it after optimized re-etching condition. The observation locations of inverted pyramid structure with five layers of kirigami graphene for TEM observation. Raman mapping of $\mathrm{I}_{2 \mathrm{D}} / \mathrm{I}_{\mathrm{G}}$ for kirigami and unpatterned graphene transferred onto the inverted pyramid structure. Scheme of hybrid PV cell with kirigami graphene and the energy band diagram of the device. PV characteristics of hybrid PV cell with and without 2-layer of unpatterned graphene. 


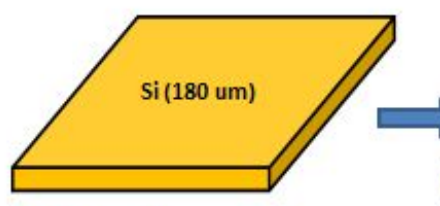

Polish silicon wafer with $\mathrm{NaOH}$

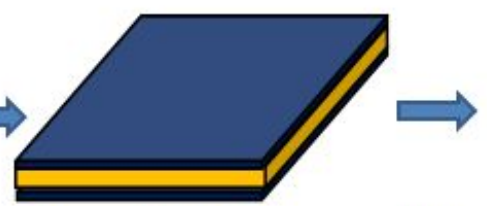

Deposit $\mathrm{SiO}_{2}$ on both sides with PECVD (100nm)

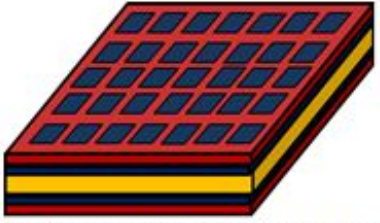

Spin photo resistance on both sides and top side with pattern

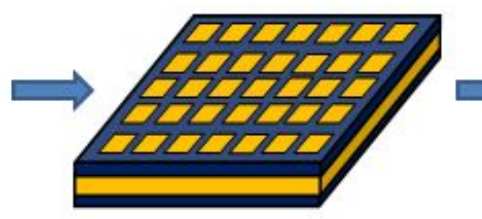

Dip BOE to remove $\mathrm{SiO}_{2}$

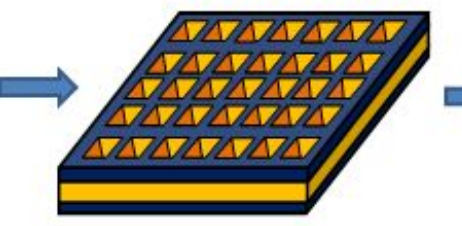

Etch the inverted pyramid with

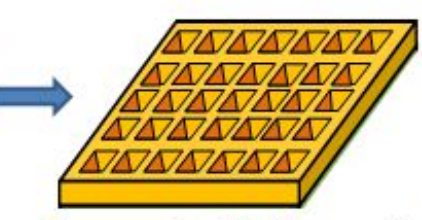

Remove both sides oxide

TMAH

Figure S1 The procedures to prepare the inverted pyramid array.

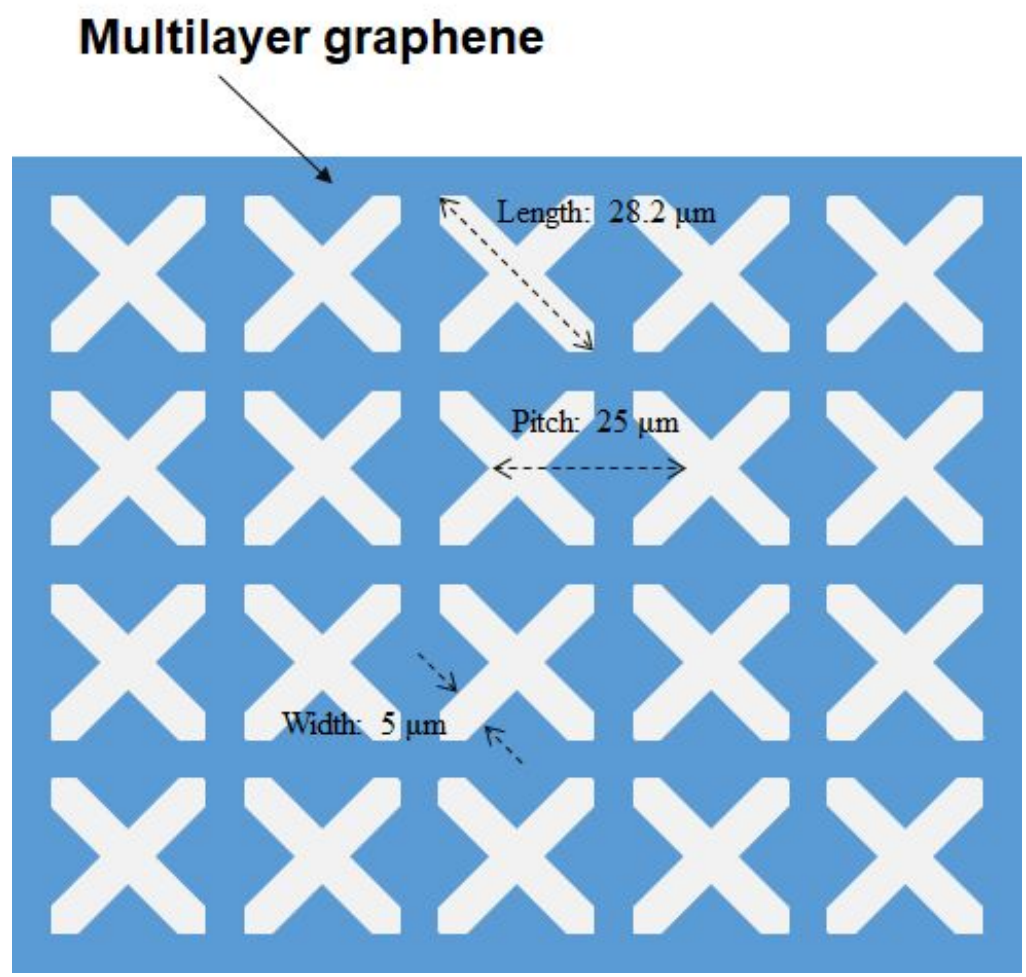

Figure S2 Scheme of kirigami pattern with cross arrays 
(a) Hybrid PV cell Top-view

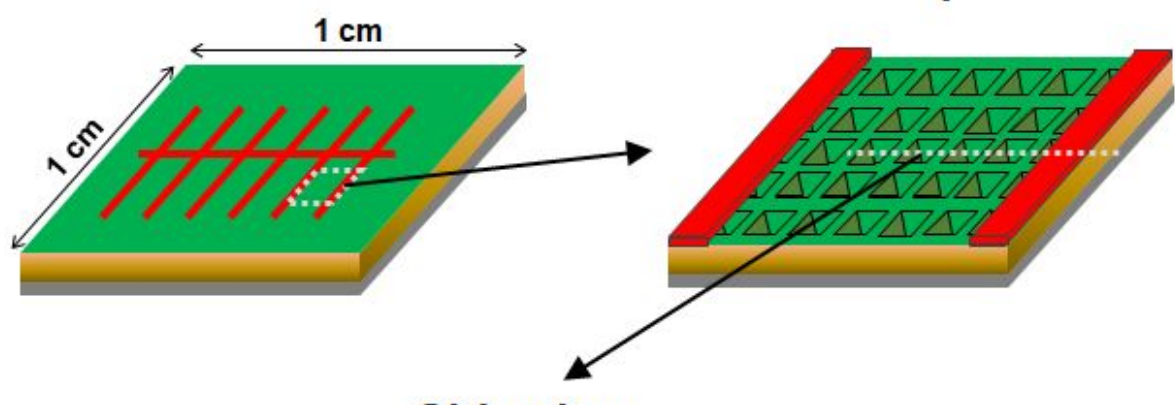
Side-view

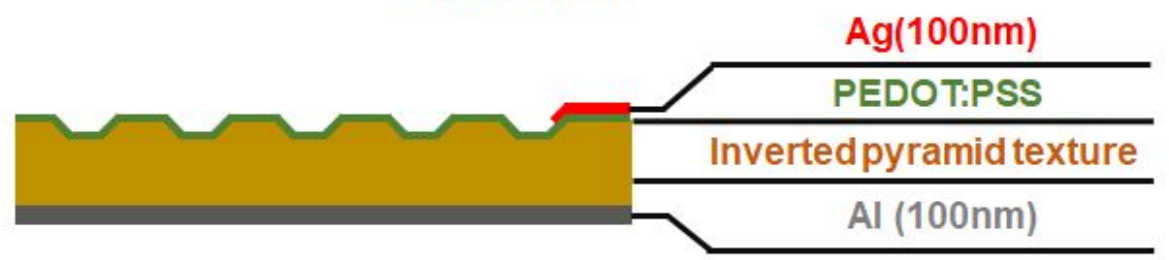

(b)

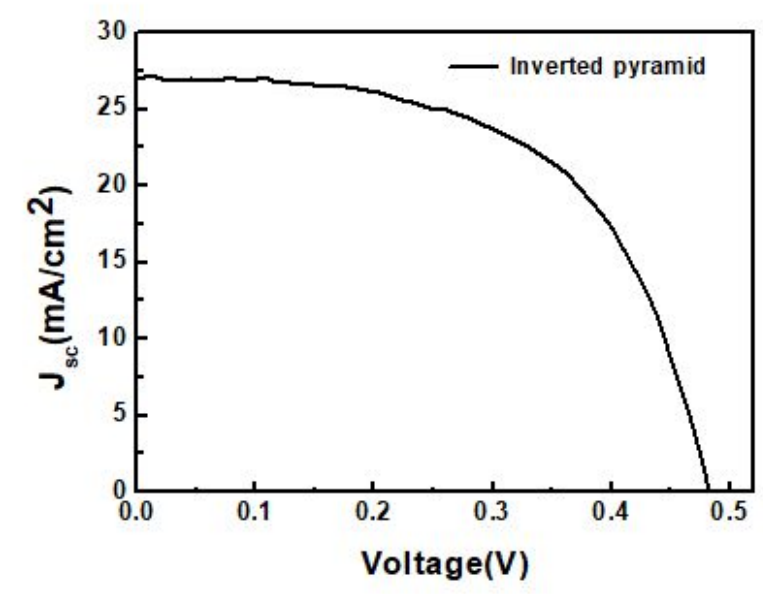

Figure S3 (a) Scheme and (b) $J-V$ curve of hybrid PV cell with inverted surface texture.

Table S1 PV characteristics of hybrid PV cells with inverted pyramid surface texture

\begin{tabular}{|c|c|c|c|}
\hline$V_{\text {oc }}(\mathrm{V})$ & $J_{\mathrm{sc}}\left(\mathrm{mA} / \mathrm{cm}^{2}\right)$ & FF & PCE (\%) \\
\hline 0.48 & 27.04 & 57.7 & 7.52 \\
\hline
\end{tabular}


(a)

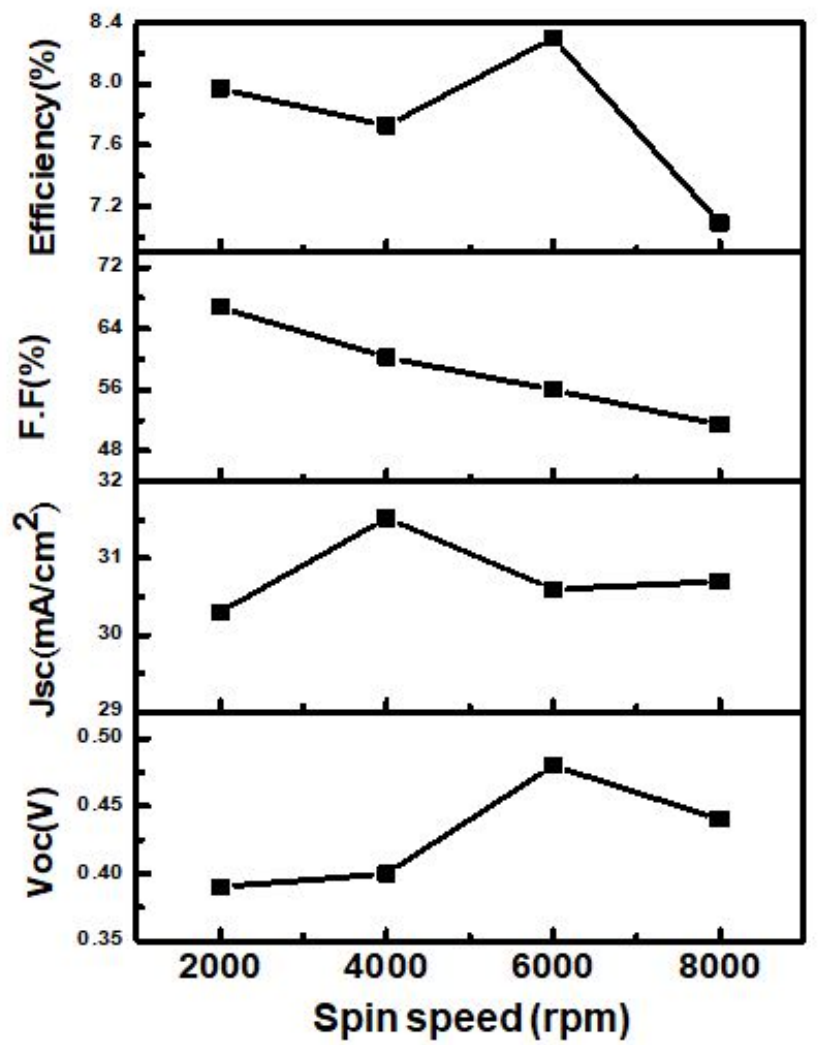

(b)

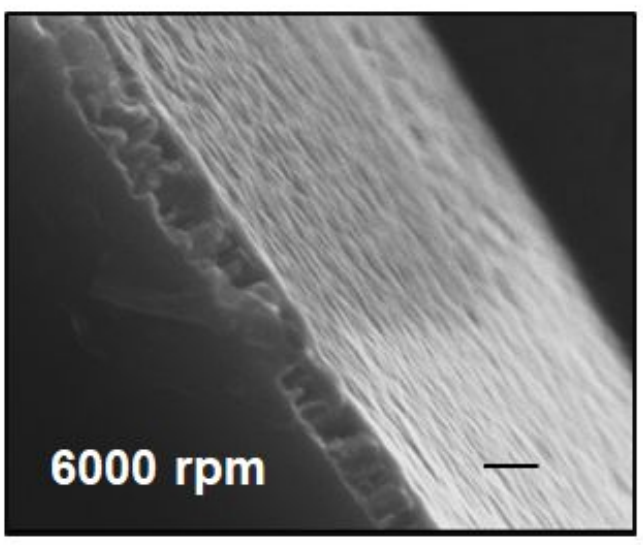

Figure S4 (a) PV characteristics of hybrid PV cell with inverted pyramid/SiNWs surface texture coated with PEDOT:PSS at different spin speeds; (b) SEM image of PEDOT:PSS coated on inverted pyramid/SiNWs at spin speed of $6000 \mathrm{rpm}$. The scale bar is $200 \mathrm{~nm}$. 
(a)

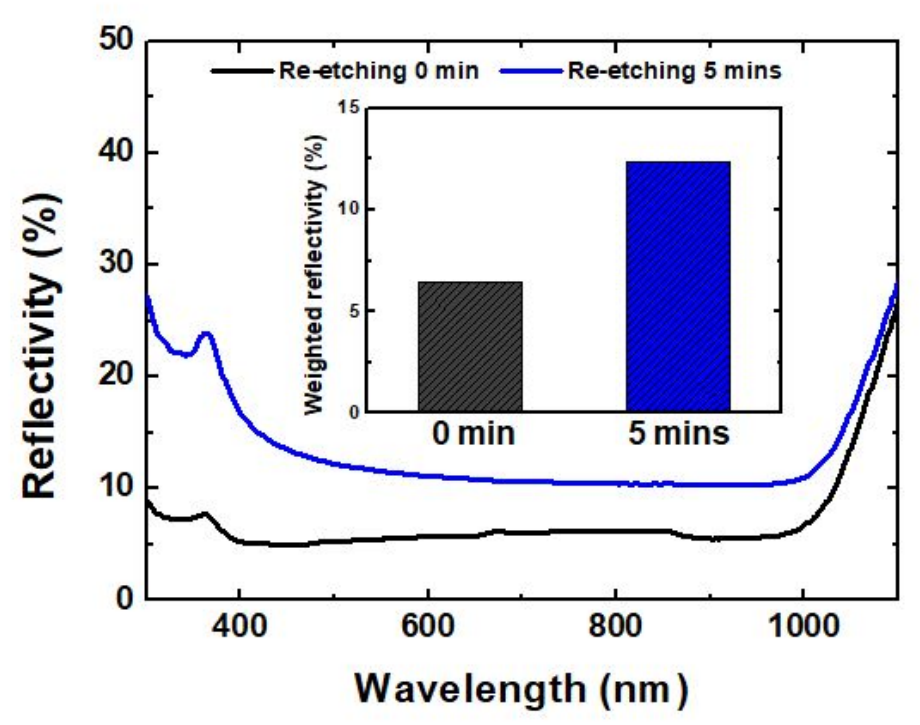

(b)

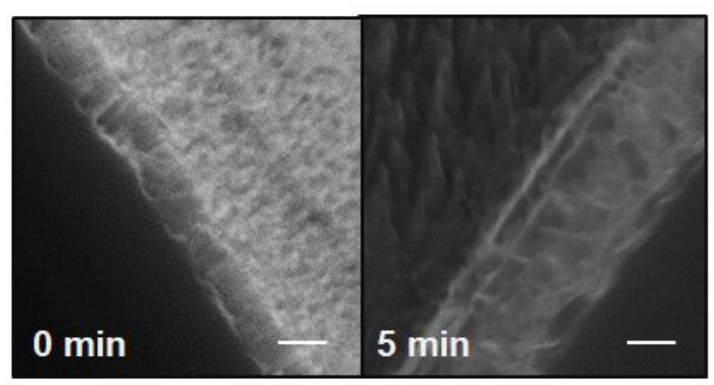

(c)

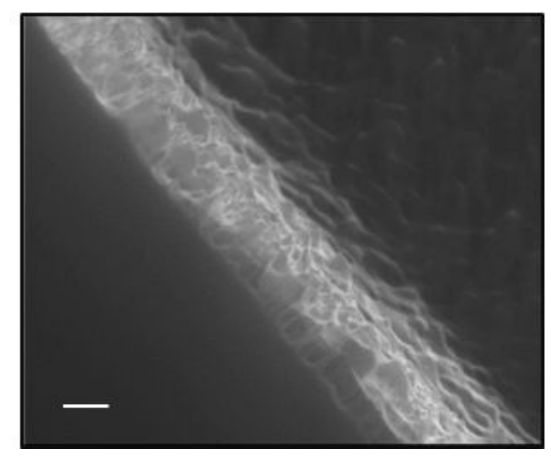

Figure 5S (a) Reflectivity spectrum of inverted pyramid/SiNWs before re-etching and after 5 min re-etching time. Inset shows the weighted reflectivity; (b) SEM images of inverted pyramid/SiNWs before and after re-etching; (c) SEM image of PEDOT:PSS coated inverted pyramid/SiNWs after 5 min re-etching. The scale bar is $200 \mathrm{~nm}$. 
(a)

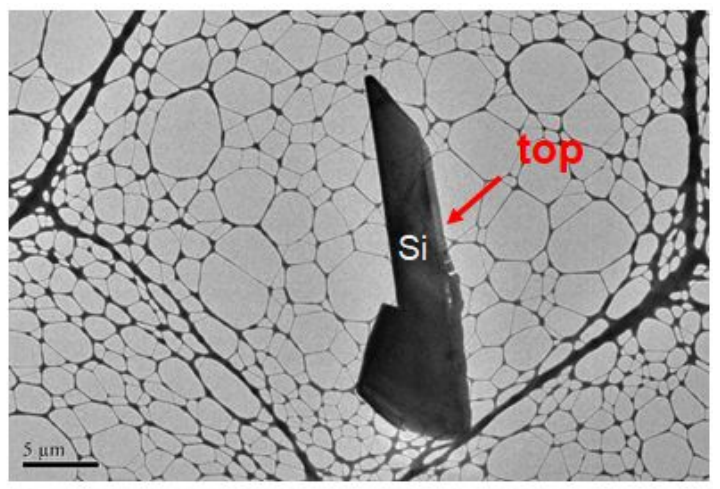

(b)

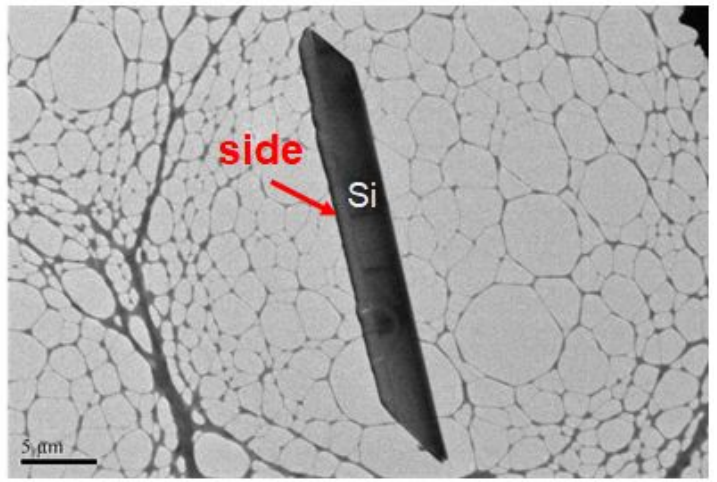

(c)

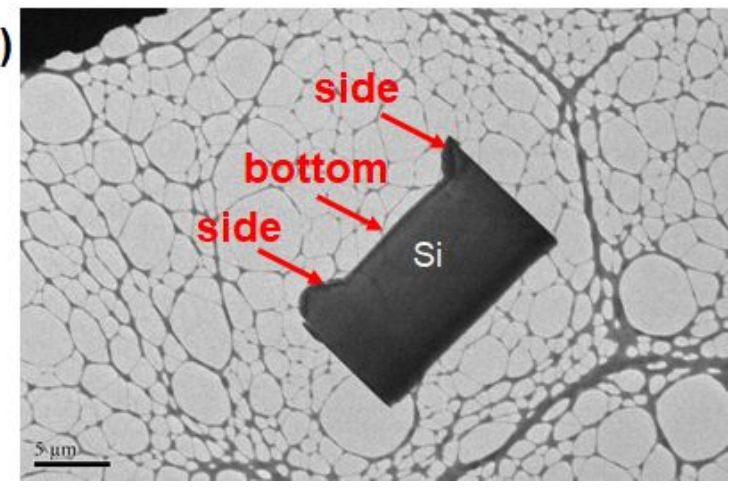

Figure S6 Three TEM samples prepared by focus ion beam etching for locations (a) top, (b) side, and (c) bottom locations. The scale bar is $5 \mu \mathrm{m}$. 


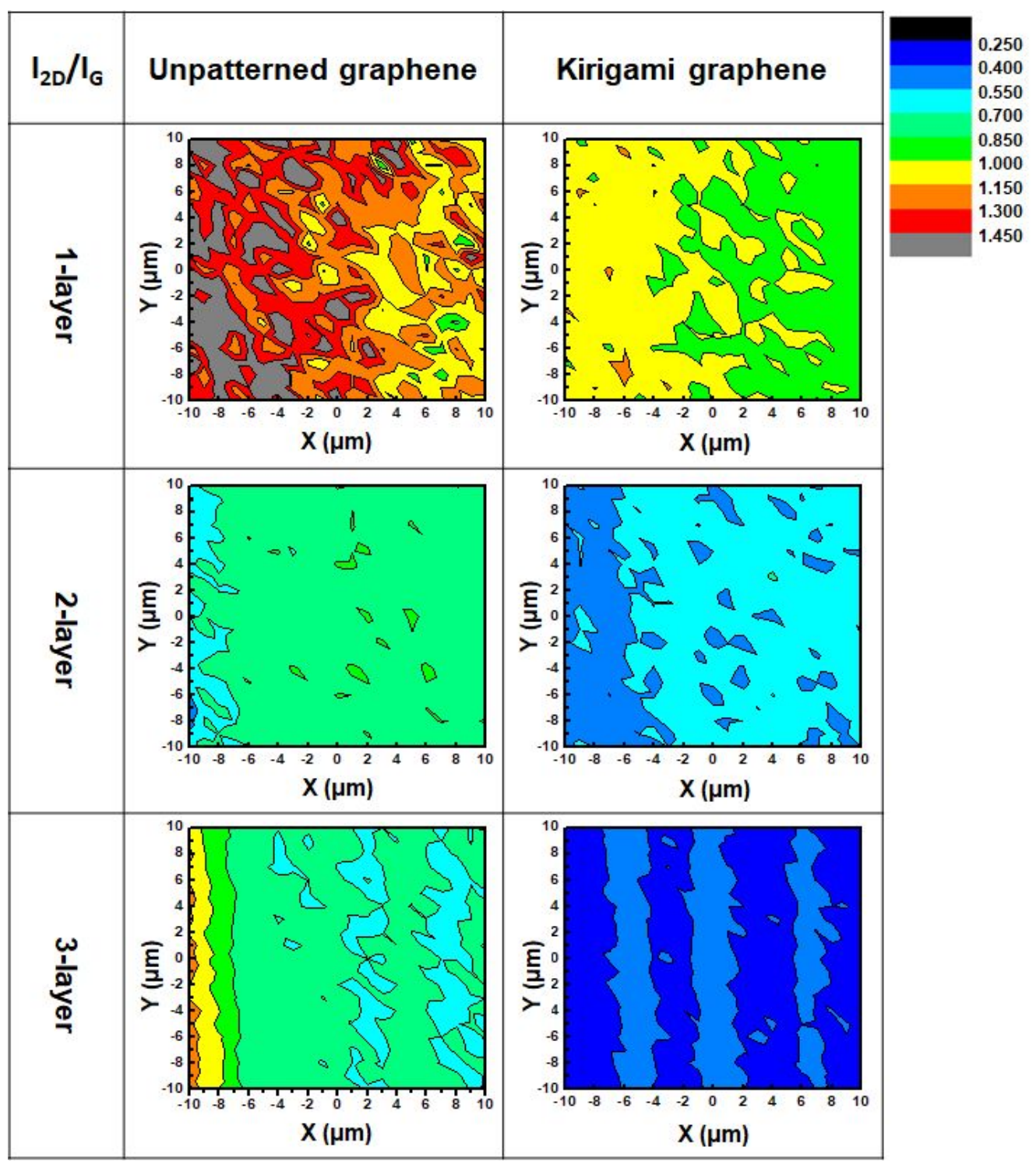

Figure S7 Raman mapping $\mathrm{I}_{2 \mathrm{D}} / \mathrm{IG}$ of unpatterned and kirigami graphene on inverted pyramid Si substrate with 1-3 layers graphene. 
(a) Hybrid PV cell

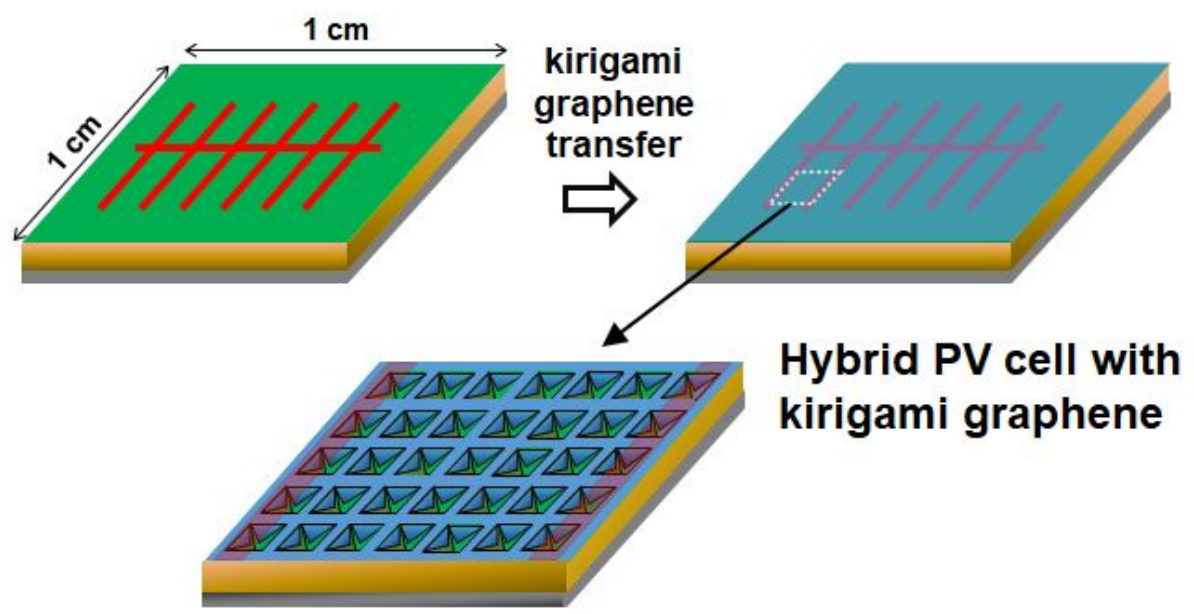

(b)

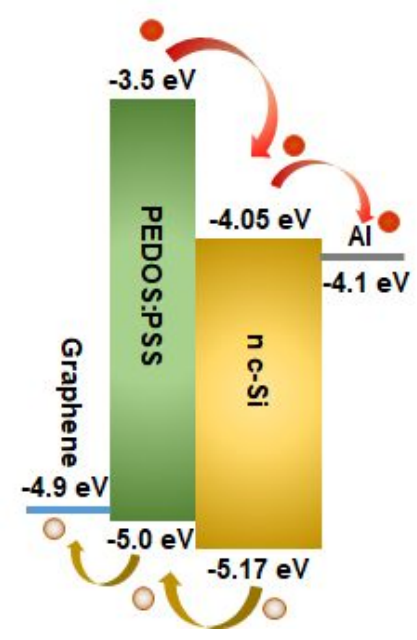

Figure S8 (a) Structure and (b) energy band diagram of hybrid PV cell with graphene 


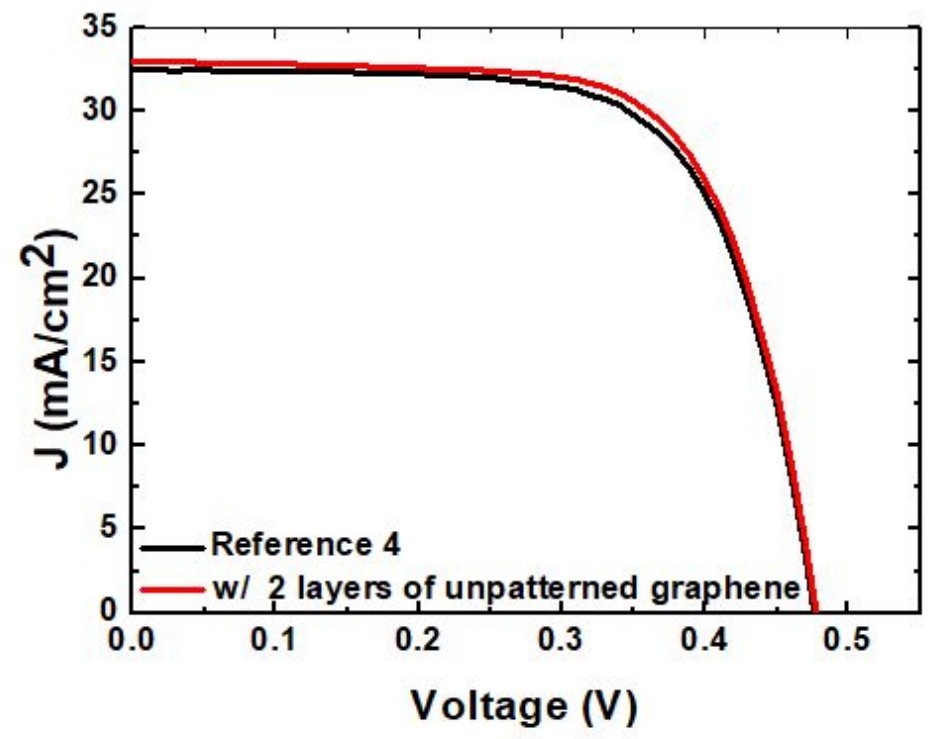

Figure S9 $J-V$ curves of Reference 4 vs with 2 layers of unpatterned graphene.

Table S2 PV characteristics of hybrid PV cells with 2 layers of unpatterned graphene and its reference.

\begin{tabular}{|c|c|c|c|c|c|c|}
\hline \multirow{2}{*}{ set } & PV cells & $\begin{array}{c}\mathbf{V}_{\text {oc }} \\
(\mathbf{V})\end{array}$ & $\begin{array}{c}\mathrm{J}_{\text {sc }} \\
\left(\mathrm{mA} / \mathrm{cm}^{2}\right)\end{array}$ & $\begin{array}{c}\text { FF } \\
(\%)\end{array}$ & $\begin{array}{c}\text { Efficiency } \\
(\%)\end{array}$ & $\begin{array}{c}\text { Efficiency } \\
\text { Enhancement } \\
(\%)\end{array}$ \\
\hline \multirow{2}{*}{4} & Reference 4 & 0.47 & 32.45 & 68.17 & 10.53 & \multirow{2}{*}{$2.8 \%$} \\
\cline { 2 - 6 } & w/ 2-layer G & 0.47 & 32.98 & 68.75 & 10.83 & \\
\hline
\end{tabular}

